\title{
Achados Histeroscópicos em Mulheres na Pós-menopausa com Diagnóstico de Espessamento Endometrial por Ultra-sonografia Transvaginal.
}

\author{
Hysteroscopic Findings in Postmenopausal Women with Endometrial \\ Thickening Diagnosed by Transvaginal Ultrasound.
}

Adriana Bittencourt Campaner, Sebastião Piato, Paulo Ayrosa Galvão Ribeiro, Tsutomo Aoki, Ricardo da Fonseca Nadais, Roberto Adelino de Almeida Prado

\begin{abstract}
RESUMO
Objetivo: correlacionar espessamento endometrial diagnosticado por ultra-sonografia com os achados histeroscópicos, em mulheres na pós-menopausa.

Métodos: foi realizado estudo transversal com avaliação histeroscópica em 121 mulheres na pós-menopausa, com diagnóstico de espessamento do endométrio por ultra-sonografia transvaginal. Das pacientes incluídas, 23 (19\%) recebiam diferentes esquemas de hormonioterapia e 98 não referiam uso de reposição hormnonal.; 55 pacientes queixavam-se de sangramento por via vaginal e as restantes apresentavam-se sem esta condição. Os exames endoscópicos foram realizados ambulatorialmente, utilizando-se histeroscópio rígido de $4 \mathrm{~mm}$. Para a distensão da cavidade uterina empregou-se gás carbônico $\left(\mathrm{CO}_{2}\right)$. Biópsia foi praticada em todas as pacientes, com auxílio de cureta tipo Novak, de $3 \mathrm{~mm}$, e o material obtido submetido a estudo histopatológico.

Resultados: a espessura do endométrio variou entre 6 e $38 \mathrm{~mm}$, com média de 10,7 \pm 5,3 $\mathrm{mm}$. Os achados histeroscópicos foram: lesão polipóide, em 51 pacientes (42,1\%); endométrio atrófico, em 15 (12,4\%); sinéquia senil, em 15 (12,4\%); espessamento focal, em 13 (10,7\%); lesão cerebróide, em 6 (5,0\%); endométrio proliferativo, em 5 (4,1\%); muco, em 5 (4,1\%); mioma, em 4 (3,3\%); endométrio secretor, em 3 (2,5\%); hiperplasia endometrial, em 3 (2,5\%) e atrofia cística, em $1(0,8 \%)$. Observou-se correlação entre os achados histeroscópicos e os resultados da histopatologia em 30 dos 51 casos de pólipo, em 12 dos 15 de endométrio atrófico e na totalidade dos casos sugestivos de hiperplasia endometrial e de adenocarcinoma.

Conclusão: na maioria das pacientes, o exame histeroscópico revelou que não se tratava de real espessamento endometrial, mas sim de outras variedades de lesão da cavidade uterina.
\end{abstract}

PALAVRAS-CHAVE: Espessamento endometrial. Menopausa. Histeroscopia. Endométrio: carcinoma. Endométrio: investigação.

\section{Introdução}

A incidência do carcinoma endometrial vem aumentando nos últimos anos, sendo mais evi-

Departamento de Obstetrícia e Ginecologia - Faculdade de Ciências Médicas da Santa Casa de São Paulo

Correspondência:

Adriana Bittencourt Campaner

Av. Rebouças 1511, apto. 142 - Jardim América

05401-200 - São Paulo - SP

Fone: (11) 3064-2878 - Fax: (11) 223-9922 - ramal 346

e-mail: abcampaner@terra.com.br dente nos países com maior desenvolvimento socioeconômico. Sabe-se que cerca de $75-80 \%$ dos carcinomas endometriais ocorrem em pacientes na pós-menopausa ${ }^{1,2}$.

Sangramento uterino é a principal manifestação clínica do carcinoma da mucosa uterina. Do ponto de vista clínico, contudo, cabe ressaltar que grande parte das mulheres portadoras desta doença maligna do endométrio apresentam-se assintomáticas $^{1-3}$. Assim, pelo estudo de $2.964 \mathrm{mu}-$ lheres sem sangramentos por via vaginal, que foram submetidas a biópsia do endométrio, 
Korhonen et al. ${ }^{4}$ constataram a presença de carcinoma endometrial em 0,07\%.

Por tais motivos, o estudo do endométrio passa a ter importância relevante em todas as mulheres que se encontram no climatério pós-menopausa e na senectude, com o propósito de detectar eventuais lesões precursoras e carcinomas iniciais da mucosa uterina. Tendo em vista as limitações da propedêutica clínica, é necessária a utilização de métodos complementares adequados.

A ultra-sonografia (USG) transvaginal é, indubitavelmente, o método pelo qual se deve iniciar a propedêutica do endométrio e da cavidade uterina, seja para orientar o diagnóstico em mulheres com sangramento genital ou para rastrear aquelas assintomáticas ${ }^{5-9}$.

Alguns estudos, entretanto, têm evidenciado que nem sempre existe correlação entre o achado de espessamento da mucosa uterina, obtido pela USG transvaginal, com as lesões existentes na cavidade uterina. Assim, em parte significativa das pacientes menopausadas, com eco endometrial espessado, verificou-se que as verdadeiras anormalidades intracavitárias consistiram em pólipo endometrial, sinéquia intrauterina, mioma submucoso ou líquido intracavitário, especialmente muco ${ }^{9-14}$.

Atualmente, o método propedêutico disponível mais apropriado para a avaliação da cavidade uterina em mulheres com suposto espessamento endometrial, sejam elas assintomáticas ou com sangramento vaginal, é a histeroscopia ${ }^{8,15-17}$.

$\mathrm{O}$ aperfeiçoamento da técnica, associado ao desenvolvimento de equipamentos mais modernos, como sistemas ópticos mais finos e utilização de aparelhos de vídeo acoplados, tornaram a histeroscopia método disponivel para utilização ambulatorial, dispensando anestesia e dilatação do canal cervical. O método é de fácil realização por profissionais habilitados e permite análise pormenorizada da cavidade uterina ${ }^{8,9,14,18,19}$.

O presente trabalho teve como objetivo correlacionar o aumento da espessura do eco endometrial em mulheres na pós-menopausa, detectado por USG transvaginal, com os achados histeroscópicos.

\section{Pacientes e Métodos}

No presente estudo foram analisadas prospectivamente 121 mulheres na pós-menopausa, com diagnóstico de espessamento endometrial pela ultra-sonografia (USG) transvaginal, assistidas nos Setores de Ginecologia Geral e de Climatério do Departamento de Obstetrícia e Ginecologia (DOGI) da Irmandade da Santa Casa de Misericórdia de São Paulo (ISCMSP), no período de maio de 2000 a julho de 2001 .

Foram incluídas no estudo tanto pacientes assintomáticas como aquelas com sangramento uterino, independentemente de se encontrarem ou não em uso de terapia de reposição hormonal. Os critérios de exclusão foram: câncer ginecológico previamente diagnosticado, condições impeditivas para a realização do exame histeroscópico e impossibilidade de avaliação histopatológica do material obtido por biópsia.

A idade das pacientes variou entre 42 e 82 anos (média de 59,6 anos). A menopausa se deu entre os 40 e os 60 anos, com média de 49 anos. Quanto ao tempo de pós-menopausa, oscilou de 1 a 40 anos (média de 10,5 anos). Das 121 pacientes estudadas, 23 (19\%) faziam uso de algum tipo de terapia de reposição hormonal (TRH); 55 pacientes queixavam-se de sangramento por via vaginal e as restantes apresentavam-se sem esta condição.

Os exames ultra-sonográficos foram realizados com aparelho da marca Hitachi, modelo EUB - 405, empregando-se transdutor transvaginal de 6,5 MHz de freqüência. A mensuração do eco endometrial foi realizada entre as camadas basais das paredes anterior e posterior do útero, em secções longitudinais do órgão. Considerou-se como espessado todo o eco que apresentasse $5 \mathrm{~mm}$ ou mais em sua mensuração.

A avaliação histeroscópica foi realizada ambulatorialmente, utilizando-se instrumento rígido de $4 \mathrm{~mm}$ (Endoview - Brasil), com visão panorâmica e angulação a $30^{\circ}$. Para a distensão da cavidade uterina utilizou-se o gás carbônico $\left(\mathrm{CO}_{2}\right)$. Padronizou-se o inventário da cavidade uterina em quatro tempos, que consistiram nas avaliações: panorâmica, da região cornual direita, da região cornual esquerda e aspecto geral do endométrio. Para a caracterização dos diferentes tipos de achados histeroscópicos utilizou-se a classificação de Labastida ${ }^{15}$.

Biópsia foi efetuada em todas as pacientes. Foi realizada de maneira orientada quando se tratou de lesões suspeitas, e aleatoriamente, quando não foram encontradas alterações ao exame. Em todas as ocasiões utilizou-se cureta tipo Novak de $3 \mathrm{~mm}$, acoplada a seringa descartável de $20 \mathrm{~mL}$. Os materiais obtidos foram colocados em frascos contendo solução de formaldeído a 10\% e encaminhados para estudo histopatológico.

O projeto deste trabalho foi aprovado pela Comissão Científica do DOGI e pelo Comitê de Ética em Pesquisa da ISCMSP. 
As seguintes técnicas estatísticas foram utilizadas para análise dos dados em estudo: teste de Mann-Whitney U para duas amostras independentes do eco endometrial, quando comparadas às variáveis TRH (uso ou não), sangramento espontâneo (presença ou ausência) e achados histeroscópicos e teste de proporções, para comparação entre os grupos de achados histeroscópicos e grau de espessura endometrial, com o intuito de verificar a concentração da maioria das pacientes em cada um dos grupos de interesse. Fixou-se o nível de 0,05 ou 5\% para rejeição da hipótese de nulidade, ainda que o valor de p observado esteja relatado para cada aplicação.

\section{Resultados}

A distribuição da espessura do eco endometrial variou de 6 a $38 \mathrm{~mm}$ nos 121 casos avaliados. A espessura endometrial média foi de 10,7 com desvio padrão $5,3 \mathrm{~mm}$.

A média ( \pm desvio padrão) para a espessura endometrial no grupo com sangramento genital foi de $11,8 \pm 6,7 \mathrm{~mm}$ e para o grupo sem sangramento foi de $9,9 \pm 3,7 \mathrm{~mm}$, não havendo diferença significativa no eco endometrial médio entre esses grupos de pacientes $(p=0,38)$. A espessura endometrial média para as pacientes em uso de hormonioterapia foi de $9,1 \pm 3,9 \mathrm{~mm}$ e para aquelas que não a utilizavam foi de $11,1 \pm 5,5 \mathrm{~mm}$, não havendo diferença significativa no eco endometrial médio dos grupos de pacientes em utilização ou não de TRH $(p=0,06)$.

$\mathrm{Na}$ Tabela 1 são apresentados os achados histeroscópicos nas 121 pacientes estudadas. Tais achados foram: lesão polipóide, em 51 pacientes (42,1\%); endométrio atrófico, em 15 (12,4\%); sinéquia senil, em 15 (12,4\%); espessamento focal, em 13 (10,7\%); lesão cerebróide, em 6 (5,0\%); endométrio proliferativo, em 5 (4,1\%); muco, em $5(4,1 \%)$, mioma, em 4 (3,3\%); endométrio secretor, em $3(2,5 \%)$; hiperplasia endometrial, em 3 $(2,5 \%)$ e atrofia cística, em $1(0,8 \%)$.

Entre as 51 pacientes com achado histeroscópico de lesão polipóide, o exame histológico dos materiais obtidos pela biópsia evidenciou pólipo endometrial em 30, endométrio atrófico em 7, endométrio proliferativo em 5, endométrio secretor em 1, atrofia cística em 5 e carcinoma espinocelular, adenocarcinoma e hiperplasia endometrial, respectivamente em 1 caso de cada.
Tabela 1 - Achados histeroscópicos nas 121 pacientes com espessamento endometrial diagnosticado pela USG transvaginal.

\begin{tabular}{lrr} 
Achados Histeroscópicos & $\mathbf{n}$ & $\%$ \\
\hline Lesão polipóide & 51 & 42,1 \\
Endométrio atrófico & 15 & 12,4 \\
Sinéquia senil & 15 & 12,4 \\
Espessamento focal & 13 & 10,7 \\
Lesão cerebróide & 6 & 5,0 \\
Endométrio proliferativo & 5 & 4,1 \\
Muco & 5 & 4,1 \\
Mioma & 4 & 3,3 \\
Endométrio secretor & 3 & 2,5 \\
Hiperplasias endometriais & 3 & 2,5 \\
Atrofia cística & 1 & 0,8 \\
Total & 121 & 100,0 \\
\hline
\end{tabular}

Na avaliação histológica das biópsias das 15 pacientes em que o achado foi sugestivo de endométrio atrófico, verificou-se concordância com o diagnóstico histopatológico em 12; nos três casos restantes, o endométrio era proliferativo em dois e misto em um.

Em relação aos 15 casos com achado de sinéquia uterina, o exame histológico revelou endométrio atrófico em 11, endométrio proliferativo em 3 e hiperplasia sem atipias em 1.

Em relação aos 3 casos com achados histeroscópicos sugestivos de hiperplasia houve confirmação pelo exame histopatológico. Quanto aos 6 casos com aspecto endoscópico de lesões cerebróides, verificou-se pela histopatologia tratar-se de adenocarcinoma do endométrio em todos. No que se refere aos 13 casos de espessamento focal do endométrio, constatou-se pelo exame histológico que em 2 se tratava de alterações hiperplásicas, em 5 de endométrio proliferativo, em 4 de endométrio atrófico e em 2 de endométrio secretor.

Entre as 5 pacientes em que a imagem histeroscópica foi sugestiva de endométrio proliferativo, a histologia mostrou atrofia em 2 , endométrio proliferativo em 2 e hiperplasia sem atipias em 1 . Nos 5 casos com achado de muco, o exame histológico revelou tratar-se de endométrio atrófico em 4 casos e proliferativo em 1 caso. Nos 4 casos em que a histeroscopia evidenciou miomas submucosos, os exames histológicos corresponderam a endométrios atrófico em 2, proliferativo em 1 e secretor em 1.

Para as 3 pacientes em que a imagem histeroscópica foi sugestiva de endométrio secretor, a 
histologia confirmou endométrio secretor em 2 casos e mostrou endométrio proliferativo em 1 .

Os dados expostos na Tabela 2 evidenciam que houve diferença significativa $(\mathrm{p}<0,0001)$ quanto à distribuição das lesões polipóides, em relação aos grupos de espessura endometrial, verificando-se que a maior delas aparece no grupo com espessamento endometrial igual ou superior a $8 \mathrm{~mm}$. Dos 6 casos de lesões cerebróides, 1 apresentou eco endometrial de $6 \mathrm{~mm}$ e os demais exibiram eco maior ou igual de $8 \mathrm{~mm}$. No entanto, não houve diferença significativa entre essas proporções $(p=0,341)$. A totalidade das hiperplasias endometriais foi encontrada em pacientes com eco igual ou maior de $8 \mathrm{~mm}$.

Tabela 2 - Correlação entre os achados histeroscópicos e 0 grau de espessura endometrial diagnosticado pela USG transvaginal.

\begin{tabular}{lrrrr}
\hline & \multicolumn{4}{c}{ Espessura endometrial } \\
& \multicolumn{2}{c}{$\mathbf{6}$ e $\mathbf{7} \mathbf{~ m m}$} & \multicolumn{2}{c}{$\geq \mathbf{8} \mathbf{~ m m}$} \\
Achados Histeroscópicos & \multicolumn{1}{c}{$\mathbf{n}$} & $\%$ & \multicolumn{1}{c}{$\mathbf{n}$} & $\%$ \\
\hline Lesão polipóide & 8 & 20,5 & 43 & 52,4 * \\
Endométrio atrófico & 10 & 25,6 & 5 & 6,1 * \\
Sinéquia senil & 6 & 15,4 & 9 & 11,0 \\
Espessamento focal & 5 & 12,8 & 8 & 9,8 \\
Lesão cerebróide & 1 & 2,6 & 5 & 6,1 \\
Endométrio proliferativo & 3 & 7,7 & 2 & 2,4 \\
Muco & 2 & 5,1 & 3 & 3,7 \\
Mioma & 2 & 5,1 & 2 & 2,4 \\
Endométrio secretor & 2 & 5,1 & 1 & 1,2 \\
Hiperplasias endometriais & 0 & 0,0 & 3 & 3,7 \\
Atrofia cística & 0 & 0,0 & 1 & 1,2 \\
Total & 39 & 100,0 & 82 & 100,0 \\
\hline
\end{tabular}

*Correlações significantes

\section{Discussão}

Ainda que a USG apresente inegável importância no rastreamento do carcinoma do endométrio e de suas lesões precursoras, tem-se verificado que ecos com dimensões acima daquela considerada normal, nem sempre correspondem a real espessamento da mucosa uterina ${ }^{10,11,13,20}$.

A biópsia do endométrio e a curetagem uterina foram consideradas durante longo tempo, métodos invasivos de excelência, utilizados para a obtenção de fragmentos do endométrio em $\mathrm{mu}$ lheres com diagnóstico ultra-sonográfico de espessamento do mesmo. Tem-se comprovado, entretanto, que a realização destes procedimentos pode falhar na obtenção de amostras da região afetada, principalmente em casos de pólipos endometriais, miomas submucosos, lesões hiperplásicas e carcinomas focais ${ }^{13,17,21,22}$.

A histeroscopia tem sido largamente utilizada na investigação de anormalidades da cavidade uterina nos últimos anos, com elevada acurácia para a detecção das referidas doenças. Sua alta sensibilidade relaciona-se com a facilidade na identificação das lesões endometriais, graças à magnificação das mesmas e sua visão direta. Apresenta ainda a vantagem de possibilitar a realização de biópsia, seja ela dirigida ou orientada ${ }^{9,14,17,19,23}$.

Os resultados por nós obtidos com o emprego da histeroscopia em mulheres com diagnóstico ultra-sonográfico de espessamento endometrial assemelham-se aos de outros autores. Em estudo que envolveu 35 pacientes na pós-menopausa com diagnóstico de espessamento endometrial à USG transvaginal, Sheth et al. ${ }^{10}$ encontraram predominância das lesões polipóides $(28,6 \%)$, seguidas de endométrio atrófico $(28,6 \%)$. Accorsi Neto ${ }^{20}$, realizando estudo em 58 pacientes menopausadas com eco endometrial maior ou igual a $4 \mathrm{~mm}$, demonstrou a presença de pólipo como principal fator causador do diagnóstico de espessamento em 30 casos $(51,7 \%)$. Loizzi et al. ${ }^{13}$ também apontaram os pólipos como principais lesões confundidas com espessamento endometrial. Estes autores observaram tal ocorrência em 23,2\% de 155 pacientes nas mesmas condições.

Para que não ocorram discrepâncias entre o achado histeroscópico e o exame histopatológico, é de fundamental importância que a biópsia da lesão seja realizada pelo método mais apropriado, isto é, a técnica dirigida. Na literatura, as taxas de falhas com o emprego de biópsias pela técnica orientada variam em torno de 10 $30 \%{ }^{9,14,20,24,25}$.

Pelo fato de não dispormos de instrumental adequado para realização de biópsias dirigidas, a biópsia histeroscópica foi orientada a lesões suspeitas ou aleatoriamente, quando não foram encontradas alterações ao exame. Isto certamente explica o fato de que em apenas 30 dos nossos casos com achado de lesões polipóides $(58,9 \%)$ houve confirmação histológica de pólipo endometrial.

Ao estudar 115 mulheres assintomáticas na pós-menopausa, por meio de exame histeroscópico, utilizando o mesmo tipo de biópsia endometrial por nós empregado, Gajardoni ${ }^{24}$ demonstrou falha nesta forma de metodologia em 16 pacientes $(13,9 \%)$. Este tipo de falha esteve relacionado principalmente à presença de pólipos. Machado et al. ${ }^{14}$, ao avaliarem a acurácia da histeroscopia como 
método de estudo da cavidade uterina em pacientes na pós-menopausa com sangramento vaginal, comparando as imagens obtidas com os resultados histológicos, demonstraram que a sensibilidade e especificidade deste método foram respectivamente $85,7 \%$ e $88,7 \%$. Estes autores relacionam os baixos índices de diagnóstico, principalmente nos casos de miomas, neoplasias e hiperplasias endometriais, à utilização da técnica de biópsia orientada. Já Scavuzzi et al. ${ }^{9}$ encontraram boa concordância entre os achados histeroscópicos e histopatológicos (kappa =0,61), utilizando técnica de biópsia adequada, apresentando diagnóstico correto de 13 dos 16 casos de câncer, em pacientes na pós-menopausa com sangramento vaginal.

As demais discrepâncias entre os achados histeroscópicos e os resultados histopatológicos em nosso estudo, previamente detalhados, igualmente se deveram à prática inapropriada de biópsia orientada. Com vista à obtenção de maior sensibilidade deste método endoscópico, evitando-se equívoco diagnóstico e decorrente impacto negativo na conduta, idealmente deve-se dispor de equipamento que possibilite a realização de biópsia dirigida.

Os resultados referidos na literatura e aqueles obtidos no presente estudo mostram que, na maioria das mulheres na pós-menopausa em que o eco endometrial apresenta-se aumentado, existem lesões que não correspondem a aumento da espessura da mucosa uterina, especialmente pólipos. Demonstram, outrossim, que a histeroscopia é método de elevada acurácia na identificação de anormalidades intra-uterinas, responsáveis por imagem ultra-sonográfica de espessamento endometrial, sempre seguida de biópsia para confirmação histológica.

\section{ABSTRACT}

Purpose: to correlate endometrial thickening diagnosed by ultrasonography with hysteroscopic findings in postmenopausal women.

Methods: a transversal study with hysteroscopic evaluation was performed in 121 postmenopausal women, with endometrial thickening diagnosed through transvaginal ultrasonography. In 98 women there was no history of hormonal replacement therapy, while the remaining 23 received different types of hormone; 55 patients complained of vaginal bleeding and the remaining did not present this condition. The endoscopic examinations were performed in the outpatient clinic, using a $4 \mathrm{~mm}$ rigid hysteroscope. For uterine cavity distention carbon dioxide $\left(\mathrm{CO}_{2}\right)$ was used. Biopsy was performed in all patients, with a $3 \mathrm{~mm}$ Novak type curette, and the collected material was submitted to a histopathological study.
Results: endometrial thickening varied from 6 to $38 \mathrm{~mm}$, with a mean of $10.7 \pm 5.3 \mathrm{~mm}$. The hysteroscopic findings were: polypoid lesion in 51 patients (42.1\%); atrophic endometrium in 15 patients (12.4\%); senile synechia in 15 patients $(12.4 \%)$, focal thickening in 13 patients $(10.7 \%)$; cerebroid lesion in 6 patients $(5.0 \%)$; proliferative endometrium in 5 patients $(4.1 \%)$; mucus in 5 patients (4.1\%); myoma in 4 patients (3.3\%); secreting endometrium in 3 patients (2.5\%); endometrial hyperplasia in 3 patients $(2.5 \%)$; and cystic atrophy in 1 patient $(0.8 \%)$. Correlation between hysteroscopic findings and cytopathology was observed in 30 of 51 cases of polypoid lesion, in 12 of 15 cases of atrophic endometrium and in all cases in which the diagnosis of endometrial hyperplasia or adenocarcinoma was suspected.

Conclusion: in the majority of the patients, the hysteroscopic examinations revealed that there was no genuine endometrial thickening but rather other types of lesion in the uterine cavity.

KEYWORDS: Endometrial thickening. Menopause. Hysteroscopy. Endometrium: carcinoma.

\section{Agradecimento}

Agradecemos ao NAP-SC Núcleo de Apoio à Publicação da Faculdade de Ciências Médicas da Santa Casa de São Paulo o suporte técnico-científico à publicação.

\section{Referências}

1. Rose PG. Endometrial carcinoma. N Engl J Med 1996; 335:640-9.

2. Souza JHK, Kalil IV, Alves FMT, Sá Filho CM, Junqueira MSR, Silva HMS. Câncer do endométrio. GO Atual 2001; 10:21-8.

3. Osmers R, Volksen M, Schauer A. Vaginosonography for early detection of endometrial carcinoma? Lancet 1990; 335: 1569-71.

4. Korhonen MO, Symons JP, Hyde BM, Rowan JP, Wilborn WH. Histologic classification and pathologic findings for endometrial biopsy specimens obtained from 2964 perimenopausal and postmenopausal women undergoing screening for continuous hormones as replacement therapy (CHART 2 Study). Am J Obstet Gynecol 1997; 176:377-80.

5. Ylostalo PR. Ultrasonography of endometrium. Ann Med 1990; 22:105-8.

6. Lerner JP, Timor-Tritsch IE, Monteagudo A. Use of transvaginal sonography in the evaluation of endometrial hyperplasia and carcinoma. Obstet Gynecol Surv 1996; 51:718-25. 
7. Buyuk E, Durmusoglu F, Erenus M, Karakoc B. Endometrial disease diagnosed by transvaginal ultrasound and dilatation and curettage. Acta Obstet Gynecol Scand 1999; 78:419-22.

8. Gull B, Carlsson S, Karlsson B, Ylostalo P, Milsom I, Granberg S. Transvaginal ultrasonography of the endometrium in women with postmenopausal bleeding: is it always necessary to perform an endometrial biopsy? Am J Obstet Gynecol 2000; 182:509-15.

9. Scavuzzi A, Amorim M, Pinho Neto JS, Santos LC. Comparação entre os achados ultra-sonográficos, histeroscópicos e histopatológicos no sangramento uterino da pós-menopausa. Rev Bras Ginecol Obstet 2003; 25:229-35.

10.Sheth S, Hamper UM, Kurman RJ. Thickened endometrium in the postmenopausal woman: sonographic-pathologic correlation. Radiology 1993; 187:135-9.

11. Dubinsky TJ, Parvey HR, Gormaz G, Curtis M, Maklad N. Transvaginal hysterosonography: comparison with biopsy in the evaluation of postmenopausal bleeding. J Ultrasound Med 1995; 14:887-93.

12.Laughead MK, Stones LM. Clinical utility of saline solution infusion sonohysterography in a primary care obstetric-gynecologic practice. Am J Obstet Gynecol 1997; 176:1313-8.

13.Loizzi V, Bettocchi S, Vimercati A, et al. Hysteroscopic evaluation of menopausal women with endometrial thickness of $4 \mathrm{~mm}$ or more. $\mathrm{J}$ Am Assoc Gynecol Laparosc 2000; 7:191-5.

14. Machado MKN, Pina H, Matos E. Acurácia da histeroscopia na avaliação da cavidade uterina em pacientes com sangramento uterino pósmenopausa. Rev Bras Ginecol Obstet 2003; 25:237-41.

15.Labastida RN. Tratado y atlas de histeroscopia. $1^{\mathrm{a}}$ ed. Barcelona: Salvat; 1990.
16. Melo NR, Pinheiro W, Petti DA, Pinotti JA. Histeroscopia: o momento atual. RBM-GO 1998; 9:238- 42.

17.Loverro G, Bettocchi S, Cormio G, et al. Transvaginal sonography and hysteroscopy in postmenopausal uterine bleeding. Maturitas 1999; 33:139-44.

18. Mencaglia L. Hysteroscopy and adenocarcinoma. Obstet Gynecol Clin North Am 1995; 22:573-9.

19.Garuti G, Sambruni I, Cellani F, Garzia D, Alleva $\mathrm{P}$, Luerti M. Hysteroscopy and transvaginal ultrasonography in postmenopausal women with uterine bleeding. Int $\mathrm{J}$ Gynaecol Obstet 1999; 65:25-33

20.Accorsi Neto AC. Estudo ultra-sonográfico, histerossonográfico, histeroscópico e anatomopatológico da cavidade uterina de mulheres na pósmenopausa [tese]. São Paulo: Escola Paulista de Medicina; 1999.

21.Accorsi Neto AC, Gonçalves WJ, Mancini SN, et al. Comparação entre a histerossonografia, a histeroscopia e a histopatologia na avaliação da cavidade uterina de mulheres na pós-menopausa. Rev Bras Ginecol Obstet 2003; 25:667-72.

22.Gimpelson RJ, Rappold HO. A comparative study between panoramic hysteroscopy with directed biopsies and dilatation and curettage: a review of 276 cases. Am J Obstet Gynecol 1988; 158:489-92.

23.Widrich T, Bradley LD, Mitchinson AR, Collins RL. Comparison of saline infusion sonography with office hysteroscopy for the evaluation of the endometrium. Am J Obstet Gynecol 1996; 174:1327-34.

24.Gajardoni SHS. Avaliação do endométrio pela ultrasonografia, histeroscopia e teste do progestogênio na pós-menopausa [tese]. São Paulo: Escola Paulista de Medicina; 1998.

25.Kent ASH, Haines P, Manners BTB, Coats PM. Blind endometrial biopsies: insufficient for diagnosis in women with intrauterine pathology. Gynaecol Endosc 1998; 7:273-8.

Recebido em: 5/11/2003 Aceito com modificações em: 9/12/2003 